\title{
Tired of slow internet? Lasers to the rescue
}

\author{
Joveria Baig \\ Department of Electrical and Electronics Engineering , UCC
}

Before you marry a person you should first make them use a computer with slow Internet to see who they really are. (Will Ferrell)

\section{Why do we need faster internet?}

The Internet has made long distances almost transparent, allowing people living in different parts of the planet to communicate in an instant. With the advent of internet in developing countries, researchers all over the world have been able to collaborate to find cures for deadly diseases prevailing in most developing nations. The average number of internet users increased from 16 million users in 1995 to 3079 million users at the end of the year 2014 and is anticipated to grow exponentially over the next decade. This increased dependence on technology also results in an increased need for faster internet.

Let's illustrate this with an example. Imagine you are a surgeon in Southern Africa and you are about to operate on a child having a potentially fatal disease. It is critical to perform the surgery at the earliest opportunity. However, there are complications involved, therefore, you decide to consult a senior surgeon living in another part of the world who has dealt with many such complications before. You turn on the video conference software to start your conversation. Unfortunately, everyone else in your building is using the internet at the same time for their ongoing research. A few seconds into your all-important video call, the video begins to deteriorate, as illustrated in Figure 1, and eventually completely vanishes. You have just suffered from a slow internet connection. Before trying to find a solution to this problem, let's first understand how the internet works.

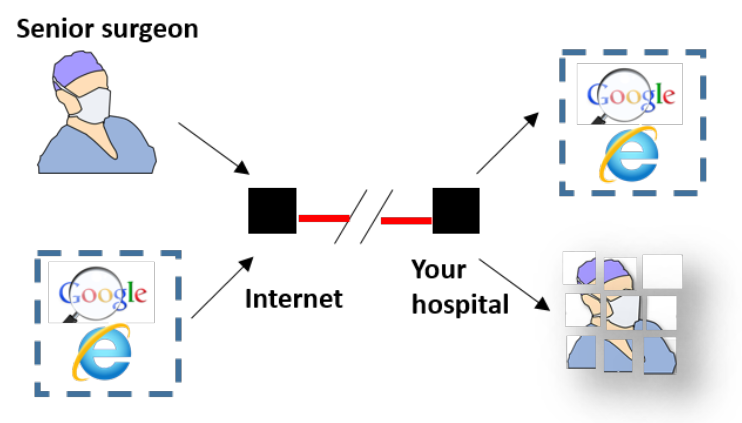

Figure 1: Simplified layout of internet framework illustrating deterioration of senior surgeon's video quality due to slow internet connectivity. Source: Joveria Baig 


\section{How does the internet work?}

During childhood, you might have made a telephone using two match boxes or baked bean cans and a span of thread. The internet nowadays is a highly advanced ultra-modern version of this telephone. There are various ways in which information travels from one end to the other. Many years ago, a wire cable was used such as in landline phones to send your voice from home to the nearest telephone exchange. With upcoming technology, it was then possible to send information in air without the need for wires, such as in cellular phones. Another mode of very fast and effective communication which has revolutionized the internet industry is the use of light to send information down a transparent pipe, known as a fiber optic cable, from one end of the globe to another. An optic fiber is a strand of glass with an exceptionally small diameter, up to ten times thinner than the human hair, which is capable of transporting up to millions of telephone calls every second. The secret to the success of optic fibers is light, which travels in these fibers at very high speeds. When you switch on a bulb in your room, you do not see light rays travelling down from the bulb to the floor; it happens so quickly that your nervous system is too slow to even register it. To give it a perspective, it takes only 1.3 seconds for light from the moon to reach the Earth! This property of light makes it an excellent carrier for data in data transmission over long distances.

So, the modern internet works like this: you send a video from your computer to a friend living in another part of the world, your computer releases electrical signals which go directly to a light source. Accordingly, a special sequence of light pulses corresponding to the video is produced and sent down an optic fiber. At the receiving end, these light pulses are converted back into electrical signals and the video you sent is decoded on your friend's computer, ready to be opened.

The choice of the light source is very important for these fibers. Due to high directionality, lasers act as excellent light sources for optic fibers primarily because they enter and leave a long distance optic fiber with minimal losses in the energy it carries. So, even with such powerful light sources, why is the internet still slow? Let's consider a simple analogy.

Imagine yourself in a situation where you are given a daunting task of transferring a million bricks from one part of the world to another within an hour. You decide to hire a red power ranger to transport these bricks for you. However, a red power ranger with his entire super powers combined needs at least a day to transport all these bricks on his own. Similar to the power ranger, laser is the super hero in the real world that is used to transport internet data (analogous to bricks) in optic fibers. But even with such great strengths, a single red colored laser (analogous to a red power ranger) has its limitations and cannot transfer so much data all at once which makes this data transfer slower than what is required. To solve this problem, internet has evolved and now uses many different colored lasers to send information along the same optic fiber. This is very similar to the red power ranger getting help from his entire team consisting of blue, yellow and green power 
rangers to transfer the bricks at a much greater speed. In summary, one colored laser, like one power ranger, carries less data in a given time. More colors of lasers together, like many power rangers, carry more data. Returning to the scenario in Figure 1, if, instead of a single wavelength laser, multiple different wavelengths were used, you would not face the problem of deteriorated video quality. This is because in this case you have a set of colored lasers (bandwidth) assigned to you that you do not share with your colleagues. Therefore, the data sent by your colleague does not affect your internet speed significantly. This is illustrated in Figure 2 which shows the internet signal flow from your hospital to the senior surgeon in the other part of the world. This idea of using multi-colored lasers is one of the main reasons why internet is much faster today than it was ten years ago.

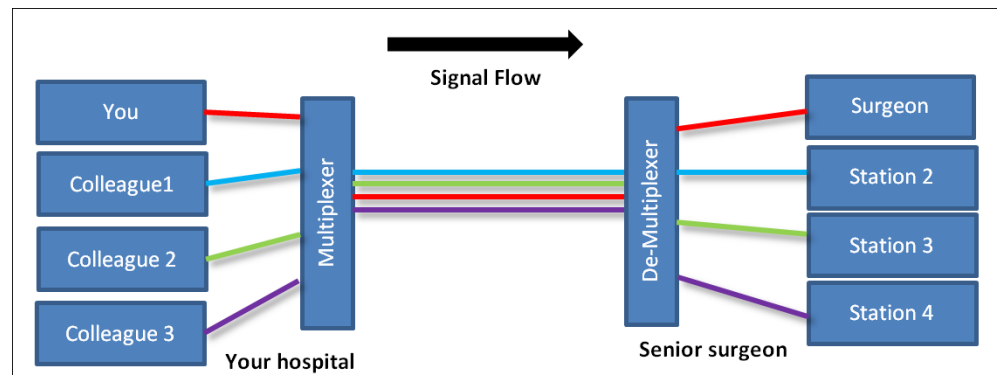

Figure 2: Simplified layout of internet framework in the case where different coloured lasers (red, blue and green as shown) are used. Source: Joveria Baig

However, with increasing demand for internet, there is a persistent need to continue to achieve faster internet. There are several ways to achieve this. One possible way is to increase the amount of data that a single colored laser can carry at a given time. Another approach is to increase the number of fiber optic cables. A third method is to introduce lasers of finely separated colors, for example, different shades of red, along with different shades of blue, etc. This latter approach, also known as wavelength division multiplexing (WDM), is the main focus of my PhD. Specifically, I am working on the design, implementation and testing of Integrated Tunable Lasers to send information down the fiber. The words tunable and integrated are crucial to my research, and will be explained in the next section.

\section{Integrated tunable lasers}

A tunable laser is one whose wavelength, or color, can be adjusted or tuned to any value. In order to understand how integrated tunable lasers are implemented, let's first understand the basic principle behind the use of a Tunable laser. On a bright sunny day when the sky is filled with droplets, we usually observe a rainbow in the sky. This rainbow is an excellent demonstration that visible light which appears to be a single white color is actually composed of many different wavelengths. These lights of different colors can be made to shine together as visible white light but can also be separated into its different color components, just as in the case of a rainbow. Lasers, on the other hand, have a property 


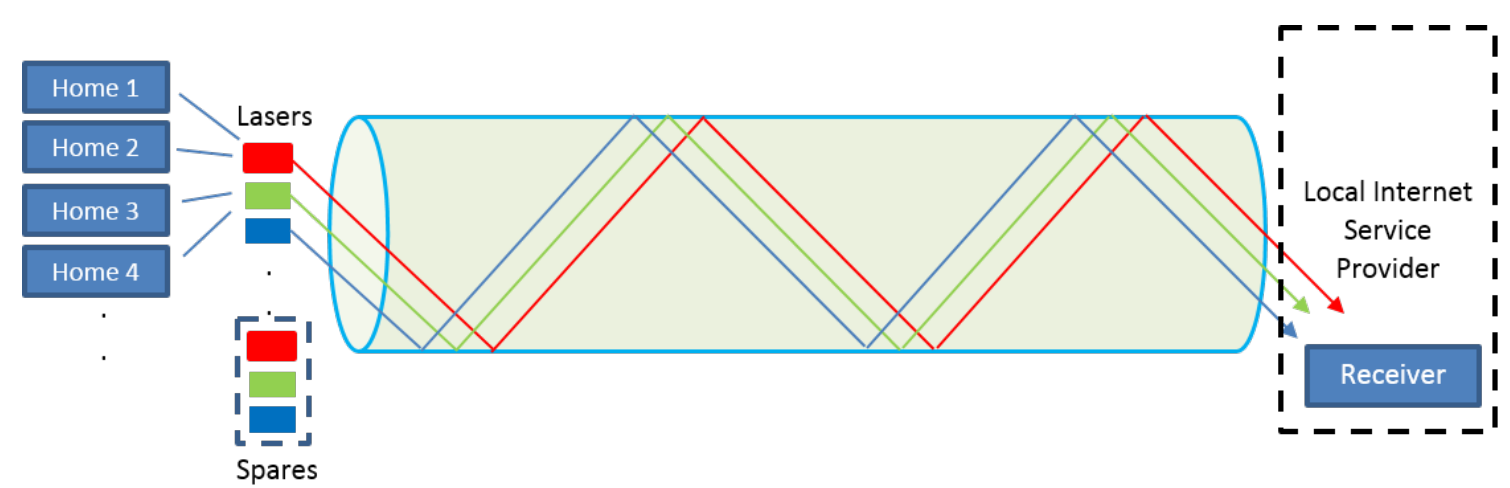

Figure 3: Simplified layout of Wavelength Division Multiplexing (WDM) infrastructure used in communication. Source: Joveria Baig

of being monochromatic in nature. Monochromatic means that inherently a single laser would have a single color. This is different from sunlight which is not monochromatic as it consists of many different colors of light. Despite the monochromatic nature of lasers, different colored lasers can be made to travel together in single optic fiber at the same time, each of them carrying different data. Although the beam altogether appears to be of a single color, data carried by the different colored lasers do not interfere with each other and can be readily de-coded at the receiver. This technique of sending different colored lasers down a single fiber increases the data carrying capacity of optic fibers. In other words, this means faster internet.

To understand the importance of tunable lasers, let's consider a simple example illustrated in Figure 3. Suppose 80 lasers of different colors (wavelengths) are used to send information along the same optical fiber. These lasers are carrying information that is transmitted from each home. For this system, another 80 lasers are placed as spare lasers in case there is a failure in the operational ones. In addition, 80 more receivers are placed to receive these spare lasers. This increases the total cost of the system significantly. A tunable laser, however, is a more cost effective solution. A single tunable laser can act as a substitute for many different colored lasers, thereby reducing the need for spare ones.

Let's return to the analogy in Figure 3 which shows that data from Home 1 and Home 2 are sent only via red laser. The data from Home 3 and Home 4, on the other hand, is sent via only the green laser. Now, imagine you are living in Home 1 and you are using internet extensively for streaming video or browsing the internet. Your friend who is living in Home 2 has an important video conference going on at the same time. So you and your friend together are requesting and sending more data over the internet which the red laser cannot carry simultaneously. Hence, it takes longer to send and receive the required data via the internet. As a result, you suffer from slow browsing speeds in Home 1 and your friend experiences lags in his conference call in Home 2. Suppose that people living in Home 3 and 4 have less data requirements, which means there is no data transfer via the green laser. In this case, red laser is over-loaded with data while the green laser is 
underutilized.

My research helps to solve the above mentioned problem. My work involves design and fabrication of tunable lasers which can quickly and readily be tuned to any chosen wavelength or channel. If a tunable laser is used instead in Home 1 or 2, it could now easily tune to green color and re-distribute the load on both red and green channels. For example, the laser in Home 1 could send data via red laser while the laser in Home 2 can tune to green laser and send data via the green channel, thus avoiding overload of a single wavelength channel. This increases the total amount of data that can be sent simultaneously and allows full use of the data carrying capacity of the optic fiber, making it a far better alternative than using fixed wavelength lasers. I will be working towards designing and implementing these lasers and to be able to increase the amount of colors/ wavelengths that a single laser can tune to. Another key aspect of my $\mathrm{PhD}$ is the integration of these lasers into tiny packages.

\section{Why is integration important?}

The recent advances in manufacturing technology has allowed us to fabricate structures in the range of nanometers. To visualize this size, if a doughnut in this world was equal to the size of the country New Zealand, then a nanometer sized object would appear to be of the size of a football. Fabricating devices down to such small dimensionality and yet achieving the desired functionality is one of the biggest wonders of the electronic industry. Thus, by using these techniques, the tunable lasers described above can be manufactured to very small dimensions. An illustration is shown in Figure 4 which shows five lasers on a chip the size of the tip of a finger nail. This small size means less material is required to engineer these devices making it cost effective and portable. With power loss minimal at this size, these devices are energy efficient. All these factors make these tiny devices more environment friendly and much cheaper than if they were produced in large sizes, allowing large scale usability. In addition, during my research, I will be concentrating on integrating these tunable lasers with other components that are required to send and receive data and to achieve it with minimal possible power losses.

\section{Internet in the future}

Internet has proved to be enormously valuable in providing far reaching economic and social benefits globally. While internet access has been nearly ubiquitous in developed economies (nearly 82 percent in 2014), a vast majority of 4.3 billion out of world's 7 billion population still stay unconnected to the internet. Most of these people belong to developing countries where internet has tremendous potential to transform the resource based economy into a knowledge based one. The advent of internet in these countries can help save lives of millions by improving medical facilities, improving the quality of 


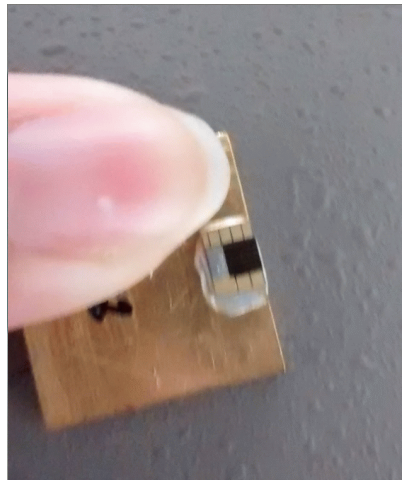

Figure 4: Comparison of the size of integrated lasers with the tip of a finger nail. The chip has five lasers on it. Source: Joveria Baig

lives of the impoverished and creating employment opportunities in areas with minimal infrastructure. But extending internet access to the vast majority of the urban poor requires a much greater bandwidth (data transfer capacity) than what is available today. Integrated tunable lasers will prove to be an excellent solution to achieve this purpose by reducing cost, size and energy consumption significantly. With the arrival of this technology, users will be able to transfer ten times more data at the same time without having to pay significantly more.

I would like to thank my supervisor Mr. Brian Corbett for his advice, guidance, and support. Acknowledgement to Science Foundation Ireland for funding this project. 